\title{
WATER VAPOR RADIOMETRY AT THE ONSALA SPACE OBSERVATORY FROM 1980 TO 1987
}

\author{
J. M. Johansson, G. Elgered, and B. O. Rönnäng \\ Onsala Space Observatory \\ S-43900 Onsala \\ Sweden
}

\begin{abstract}
Variations in atmospheric water vapor are difficult to correct for in geodetic, astrometric, and mm-wave astronomy VLBI. The use of a water vapor radiometer (WVR) has given promising results. The algorithm which relates the sky brightness temperatures observed by the WVR to the delay caused by atmopheric water vapor is discussed. Examples of WVR measurements made at the Onsala Space Observatory from 1980 to 1987 are presented.
\end{abstract}

\section{INTRODUCTION}

The main reason for the development of the water vapor radiometer (WVR) at the Onsala Space Observatory was to have a method to correct for the atmospheric delay due to water vapor during geodetic radio interferometry experiments. This delay is a major source of error when geodetic parameters such as baseline lengths are estimated.

The instrument commonly known as a WVR is a microwave radiometer which measures the emission from the sky at two (or more) well separated frequencies around the water vapor emission line at $22.235 \mathrm{GHz}$. The WVR observable at the frequency channel close to the center of the line depends on the amount of water vapor along the line of sight. The second frequency channel of the WVR is needed in order to correct for emission caused by liquid water, which occasionally can be even stronger than the vapor contribution. Since the wet delay also depends on the amount of atmospheric water vapor, we can formulate a (non-unique) algorithm that will allow us to infer the wet delay from the WVR observables.

The WVR at the Onsala Space Observatory has two channels; one centered at $21.0 \mathrm{GHz}$ and the other at $31.4 \mathrm{GHz}$. System temperatures are typically around $600 \mathrm{~K}$ at both frequencies. The bandwidth of each channel is $1 \mathrm{GHz}$ at the RF-port and typical integration times are around one second. The instrument is fully steerable and has a maximum slewing speed of $1.7^{\circ}$ per second in both azimuth and elevation. Each channel is internally calibrated by a liquid nitrogen load and a load at room temperature. External calibration is performed by elevation-scans (tip-curves). The root-mean-square (RMS) repeatability over time periods of days is believed to be better than $1 \mathrm{~K}$ in each channel.

The algorithm relating the WVR observables to the wet path delay is in no way unique. Therefore, it is possible to make many rather different approaches to the problem. Empirical parameters in the algorithm show depencence on meteorological conditions such as vertical profiles of atmospheric temperature. Algorithms can therefore be optimized to a particular site. A detailed study has been made by Johansson et al. (1987) where algorithms including as well as excluding ground meteorology parameters are derived for about 20 geodetic VLBI sites. The RMS error 


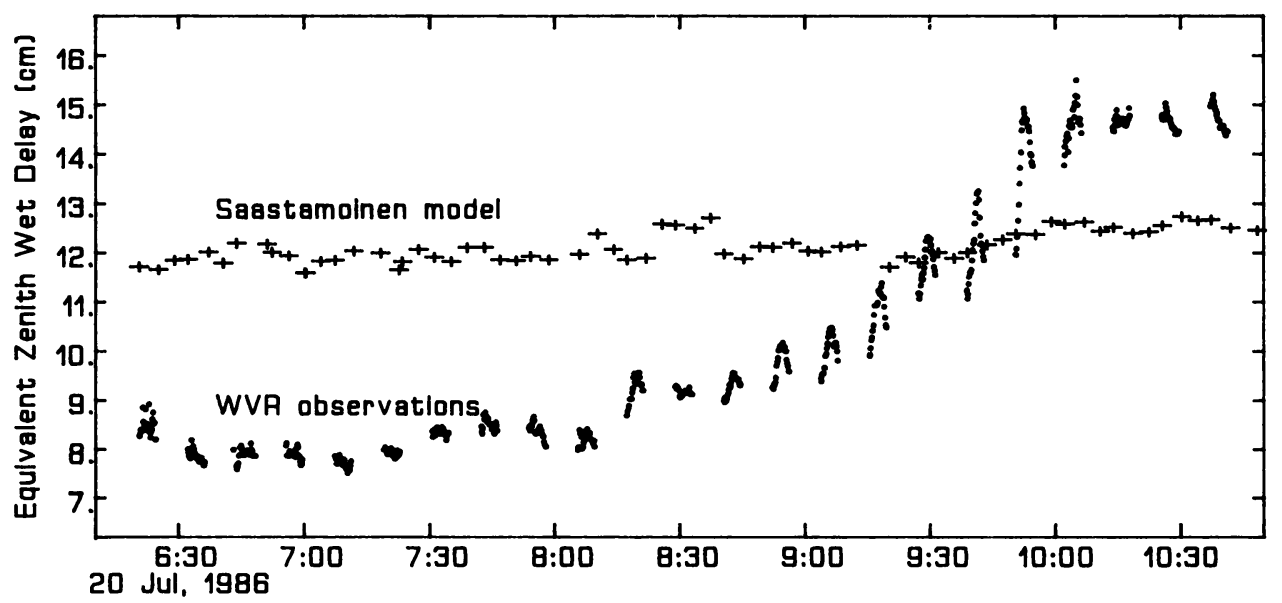

Figure 1. Equivalent zenith wet path delays measured with the WVR scanning at an elevation of $30^{\circ}$. Each data group consists of a scan from the east over the south and west and ending in the north direction. We conclude that a more humid air-mass was approaching from the west.

introduced by approximations in the algorithm is typically $2 \mathrm{~mm}$ in the zenith direction. When the algorithm is derived we assume that the attenuation due to water vapor can be perfectly calculated from in situ meteorological parameters. However, the uncertainties of these formulas are around $5 \%$, implying that the inferred wet delay has an uncertainty of the same order.

\section{RESULTS.}

Results obtained when WVR data have been used in the processing of geodetic VLBI data are reported by Elgered et al. (1987). These results suggest that it is important to improve the accuracy of formulas for the attenuation due to water vapor mentioned above.

Other results have been obtained from comparison of simultaneous WVR and radiosonde measurements at Göteborg-Landvetter Airport, 1980, and at Onsala Space Observatory, 1983. At both these occasions the RMS zenith difference of the wet delay was just below $8 \mathrm{~mm}$.

Finally, in Figure 1, we present a typically unfavourable day for the prediction of wet delays using a ground based model. The wet delay increases with almost a factor of two over a twohour period. However, the delay predicted by the model (Saastamoinen 1972) (approximately proportional to the absolute humidity at the ground) shows no variations above the $10 \%$ level.

\section{REFERENCES.}

Elgered, G., J.L. Davis, T.A Herring and I.I. Shapiro, 'The effect of the wet atmosphere on estimates of baseline lengths from VLBI,' IAU Symposium No. 129, Cambridge, MA, 1987.

Johansson, J.M., G. Elgered, J.L. Davis, 'Geodesy by radio interferometry: Optimization of wet path delay algorithms using microwave radiometer data,' Res. Rept. No. 152, Onsala Space Observatory, Chalmers Univ. of Tech., 1987.

Saastamoinen, J., 'Atmospheric correction for the troposphere and stratosphere in radio ranging of satellites,' in The Use of Artificial Satellites for Geodesy, Geophys. Monogr. Ser., 15, ed. S.W. Henriksen et al., pp. 247-251, American Geophysical Union, Washington, D.C., 1972. 